\title{
Evidence of Hofstadter's Fractal Energy Spectrum in the Quantized Hall Conductance
}

\author{
C. Albrecht, ${ }^{1}$ J. H. Smet, ${ }^{1}$ K. von Klitzing, ${ }^{1}$ D. Weiss, ${ }^{2}$ V. Umansky, ${ }^{3}$ and H. Schweizer ${ }^{4}$ \\ ${ }^{1}$ Max-Planck-Institut für Festkörperforschung, D-70569 Stuttgart, Germany \\ ${ }^{2}$ Institut für Experimentelle und Angewandte Physik, Universität Regensburg, D-93040 Regensburg, Germany \\ ${ }^{3}$ Braun Center for Submicron Research, Weizmann Institut, Rehovot 76100, Israel \\ ${ }^{4}$ IV Physikalisches Institut, Universität Stuttgart, D-70550 Stuttgart, Germany
}

(Received 17 May 2000)

\begin{abstract}
The energy spectrum of a two-dimensional electron system in a perpendicular homogeneous magnetic field and a weak lateral superlattice potential with square symmetry is composed of Landau bands with recursive internal subband structure. The Hall conductance in the minigaps is anticipated to be quantized in integer multiples of $e^{2} / h$ that vary erratically from minigap to minigap in accordance with a Diophantine equation. Hall measurements on samples with the requisite properties uncover this long searched for evidence of Hofstadter's butterflylike energy spectrum.
\end{abstract}

DOI: 10.1103/PhysRevLett.86.147

The problem of electrons confined to a plane under the simultaneous influence of a two-dimensional periodic potential and a tunable perpendicular magnetic field has long intrigued theorists and experimentalists alike. The magnetic length $l_{B}$ and lattice constant $a$ characterize the electron's motion and conspire to rich physics that culminates in the fractal single particle energy spectrum, referred to as Hofstadter's butterfly [1]. It reconciles the adverse properties of the two extremes: an energy spectrum consisting of discrete, highly degenerate Landau levels in the absence of the periodic potential and energy bands associated with Bloch waves that exhibit the translational symmetry of the lattice in the absence of the magnetic field. In the theory, the self-similar energy spectrum is obtained from both the complementary, but mathematically equivalent, limiting cases, where either the influence of the magnetic field on the strong lattice potential in the tight-binding approximation [1-3] or the influence of the modulation potential on the Landau-quantized two-dimensional electron system (2DES) [3-5] may be considered as a small perturbation. In both cases, the energy spectrum is obtained by solving Harper's equation [2]. The solution of a nearly equivalent equation that describes the propagation of microwaves in a one-dimensional array of scatterers has been verified in experiment [6].

Starting from a Landau ladder, the addition of a onedimensional sinusoidal potential, sufficiently weak to guarantee that Landau level mixing can be ignored, lifts the degeneracy and broadens the Landau levels into nonoverlapping bands [7]. The width of a Landau band with index $n$ $(n=0,1, \ldots)$ oscillates as the magnetic field is tuned and vanishes whenever the cyclotron radius, $R_{n} \equiv l_{B} \sqrt{2 n+1}$, corresponding to this Landau level is commensurate to the lattice period in accordance with the so-called flat band condition $2 R_{n}=a(\lambda-1 / 4)$, where $\lambda$ is an integer oscillation index. Periodic modulation in the perpendicular direction splits each Landau band into $p$ subbands of equal weight if the magnetic flux per unit cell of the periodic potential, $\phi=B a^{2}$, takes on a rational multiple $p / q$ of
PACS numbers: 73.20.Dx, 73.40.Hm, 73.50.-h, 73.61.-r

the flux quantum $\phi_{0}=h / e$, where $p$ and $q$ are relative primes. For values of $\phi / \phi_{0}$ with ever larger $p$ near some rational ratio $p_{0} / q_{0}$ with small $p_{0}$ the number of subbands will increase accordingly and at irrational values the number even goes to infinity. However, minigaps that exist at $p_{0} / q_{0}$ are preserved over some finite range of $\phi / \phi_{0}$, since subbands cluster into groups (as evident in Fig. 1). Ultimately, it is this property of the recursive structure of the energy spectrum that enables experimental observation of the internal Landau band structure, since only primary gaps separating clusters of subbands will survive in any physical

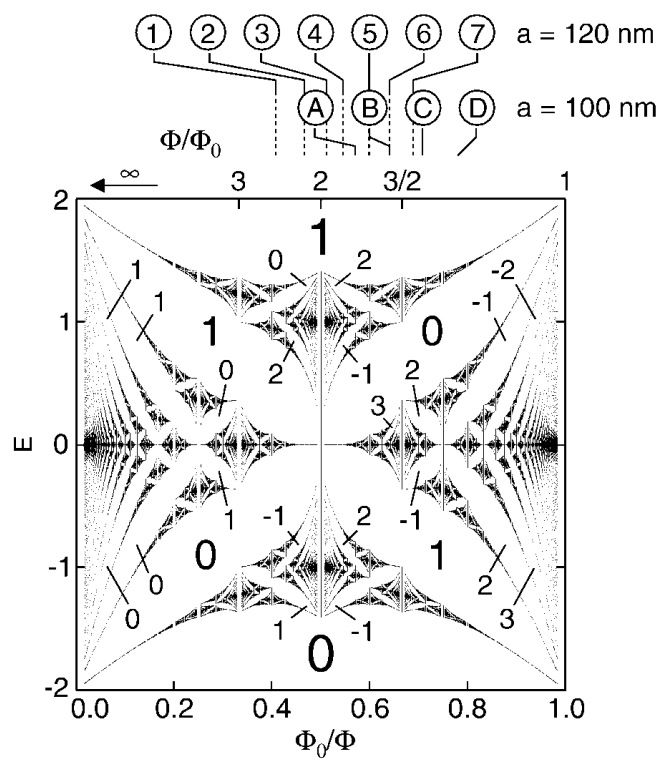

FIG. 1. The energy spectrum inside the Landau band as a function of $\phi_{0} / \phi=q / p$ (see also Ref. [12]). The energy $E$ on the ordinate is normalized to the bandwidth. The contribution of the Landau band, $\sigma e^{2} / h$, to the total Hall conductance when the Fermi energy is located within one of the primary minigaps is given in units of $e^{2} / h$. The encircled digits and letters indicate the position corresponding to SdH-maxima for the $120 \mathrm{~nm}$ and $100 \mathrm{~nm}$ lattices in Figs. 2 and 4 respectively. 
realization due to disorder-induced broadening and lattice imperfections.

For the periodic potential induced by the semiconductor crystal that hosts the 2DES, the experimentally accessible steady fields come 4 orders of magnitude short to enter the interesting regime where $\phi / \phi_{0}$ approaches values of order 1 so that clusters of subbands are separated by large gaps. Experimentalists have, in their chase for the butterfly, resorted to lateral two-dimensional superlattices patterned on top of a 2DES to impose a superstructure with increased periodicity, as originally proposed by Hofstadter [1], in order to produce artificial crystals with sufficiently large unit cells and to relax the magnetic field requirement. Still, uncovering Hofstadter's fractal energy spectrum has turned out to be a formidable task due to the stringent and, in part, contradictory requirements on the sample. Direct spectroscopic techniques of the density of states or energy spectrum are not yet feasible and attempts have focused on magnetotransport. Resolving the minigaps, despite unavoidable disorder induced broadening, compels large modulation strengths to create wide Landau bands, since their width is proportional to the potential strength, and sufficiently strong magnetic fields to ensure well-separated bands. To shift the relevant region $\phi / \phi_{0} \approx 1$ to these fields, superstructures with small lattice constants on the order of $100 \mathrm{~nm}$ are called for. However, since electric fields at the surface decay exponentially with the ratio of the 2DES depth to the period, sustaining equal modulation strength becomes excessively difficult the smaller the lattice constant and requires shallow 2DESs that are bound to suffer from increased disorder due to the reduced spacer thickness between heterointerface and dopants. Larger $B$ fields also imply an improved, undesirable screening [8], which diminishes the modulation amplitude. A complicated set of interrelated trade-offs results and the experimental window for detection shrinks to a very limited region.

Unequivocal evidence of the Hofstadter spectrum in magnetotransport should be composed of three constituents: (i) the observation of splitting of a Shubnikovde Haas $(\mathrm{SdH})$ maximum in multiple peaks attributed to the dominant energy bands and gaps in the fractal energy spectrum; (ii) a clear identification of the gaps as either a gap between Landau bands or an internal minigap within one Landau band with the aid of temperature dependent measurements; (iii) a verification of the prediction by Thouless et al. and Středa [9] on the behavior of the Hall conductance as the Fermi energy is swept through a Landau band containing minigaps. In the absence of the periodic modulation, the Hall conductance $\sigma_{H}$ is quantized between Landau levels in integer multiples of $e^{2} / h$. Contrary to classical considerations of the Hall current supported by a modulation broadened Landau band with minigaps that would suggest that each subband carries a fraction of this Hall current, Laughlin's gauge invariance argument [10] applies equally well to minigaps, irrespective of their origin. It dictates a quantized Hall conductance in integer multiples of $e^{2} / h$ even in these minigaps. Thouless and co-workers [9] derived that for Hofstadter's butterfly these multiples $\sigma$ form an irregular sequence that obeys the Diophantine equation:

$$
g=w q+\sigma p, \quad|w| \leq p / 2,
$$

where $\phi / \phi_{0}=p / q ; p, q=1,2,3, \ldots$ and $w, \sigma=$ $0, \pm 1, \pm 2, \ldots$ In this expression, $g$ is the gap index that goes from 1 to $p-1$, since there are $p$ subbands. If the Landau band with index $n$ is only partially filled and the Fermi energy is located in the $g$ th minigap, the total Hall conductance is equal to the sum of $\sigma e^{2} / h$ and $n e^{2} / h$, since $n$ bands are completely filled. For example, if $\phi / \phi_{0}$ equals $3 / 2$, the Landau band is split into three subbands. If the Fermi energy is located in the lowest minigap $(g=1)$, Eq. (1) is satisfied for $w=-1$ and $\sigma=1$, and thus the partially filled Landau band $n$ adds $e^{2} / h$ to the Hall conductance. For the second minigap $(g=2), w$ and $\sigma$ are 1 and 0 , respectively and the partially filled band does not contribute to the total Hall conductance. In Fig. 1 the internal spectrum of the Landau bands is plotted versus $\phi_{0} / \phi$ and $\sigma$ values are included for the largest gaps. The Hall conductance does not necessarily vary in a monotonous fashion as in the case without $2 \mathrm{D}$ modulation. When considering only the largest minigaps, the contribution of the Landau band to the Hall conductance exhibits either the 0-0-1-1 sequence or 0-1-0-1 sequence in units of $e^{2} / h$ when the Landau band is successively filled, depending on whether the magnetic flux per unit cell is smaller or larger than $2 \phi_{0}$, as drawn schematically later in Fig. 3(b). Moreover, since the value of the Hall conductance depends on the minigap index $g$, it discloses far more information on the internal structure of the Landau band than the longitudinal resistivity does, which simply drops to zero for every gap. Even if so, the behavior of $\sigma_{H}$ in such systems has been ignored entirely in experiment and a first glimpse of Hofstadter's butterfly was announced in Ref. [11] based on a study of the longitudinal resistance only.

Here, we report on the Hall resistance, the longitudinal resistance, and its temperature dependence to address all three points (i)-(iii). By combining these quantities, minima and even weak shoulder structures modifying the $\mathrm{SdH}$ peaks can be identified with great confidence and the interpretation can be checked for consistency. A transition from monotonous to nonmonotonous behavior of the Hall resistance, as the symmetry line of the butterfly in Fig. 1 at $\phi / \phi_{0}=2$ is crossed, is confirmed and provides clear-cut evidence for the internal Landau band structure induced by the potential of the underlying artificial crystal. This combined study opens the way to investigate more complex cases with overlapping Landau bands [12] or where interaction plays an important role [8].

The starting material for the fabrication of the lateral superlattices is a shallow GaAs/AlGaAs heterostructure with the heterointerface $48 \mathrm{~nm}$ underneath the surface. A wet chemical etch produces a mesa in the shape of a Hall bar 
with the geometry factor equal to 1 . After alloying Ohmic contacts the sample is spin coated with a 200 -nm-thick positive PMMA resist layer. A square grid of cylindrical holes with a periodicity of either 100 or $120 \mathrm{~nm}$ is defined in the resist layer with the aid of electron-beam lithography. A shadow mask evaporation covers the electronbeam patterned region of the Hall bar with a 200-nm-thick $\mathrm{NiCr} / \mathrm{Au}$ layer. It serves as a gate electrode and is periodically displaced from the 2DES by the underlying resist layer. The electron mobility of the unpatterned and patterned sample is approximately $2.1 \times 10^{6}$ and $1.5 \times$ $10^{6} \mathrm{~cm}^{2} / \mathrm{V} \mathrm{s}$ at a carrier density of $3 \times 10^{11} / \mathrm{cm}^{-2}$. Previous experiments on samples of similar design [13] have indicated that the 2D modulation originates mainly from stress or Fermi level pinning effects at the semiconductor surface, rather than from the applied gate voltage. The modulation amplitude is on the order of $0.6 \mathrm{meV}$, so that we are in the weak coupling regime [12]. The longitudinal and Hall resistance were measured with conventional lock-in techniques in a dilution refrigerator.

Figure 2 depicts the longitudinal and inverse Hall resistance [14] of the $120 \mathrm{~nm}$ lattice, where the $\mathrm{SdH}$ minima approach zero and Hall plateaus are well developed. The SdH-maxima of interest are assigned the digits 1-7 from high to low magnetic field and will be discussed consecutively. For ease of orientation, the same digits mark the approximate $\phi_{0} / \phi$ values in Fig. 1. This example was selected intentionally, since the symmetry axis of the Hofstadter butterfly at $\phi / \phi_{0}=2$ is crossed and Landau bands with an internal structure representative of the left and the right side of the butterfly are both covered. The $\mathrm{SdH}$ peak 1 between filling factor $\nu=16(0.76 \mathrm{~T})$ and $\nu=18$

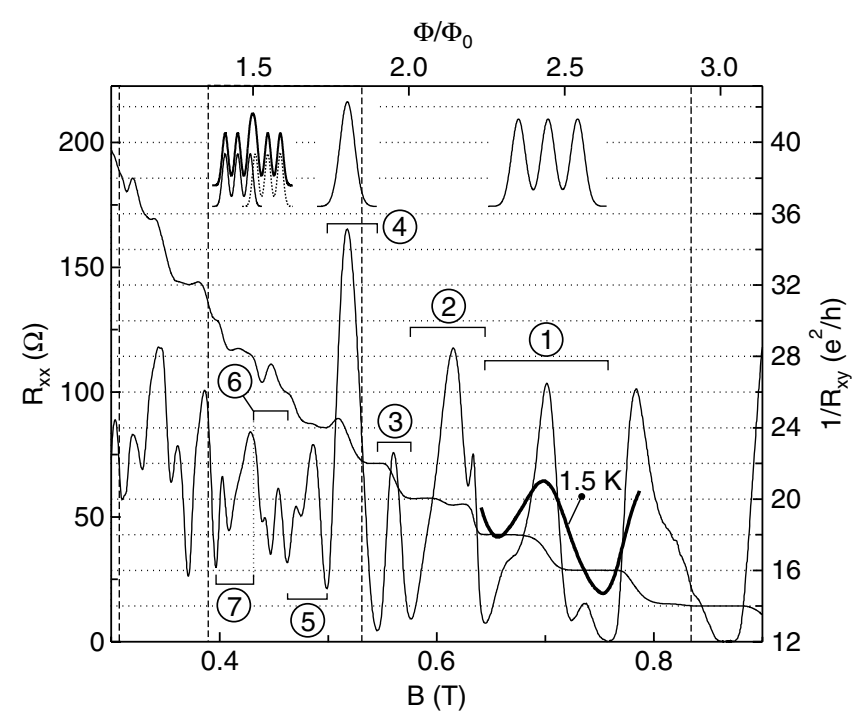

FIG. 2. The longitudinal resistance $R_{x x}$ and inverse Hall resistance, $1 / R_{x y}$, for a $120 \mathrm{~nm}$ lattice at $50 \mathrm{mK}$. The quantized values of $R_{x y}$ are drawn as horizontal dotted lines. Vertical dashed lines mark positions of vanishing Landau bandwidth. The inserts depict the resolvable minigaps. The high temperature $\left(1.5 \mathrm{~K}\right.$, bold) curve of $R_{x x}$ is shown around the SdH-peak marked 1.
$(0.64 \mathrm{~T})$ (spin splitting is not resolved yet) occurs where minibands are clustered in three main groups, as indicated schematically in the inset. As anticipated, the peak exhibits a threefold splitting, even though one of the additional minima is not fully developed yet and is only announced by a shoulder structure. The detailed temperature dependent study of $R_{x x}$ confirms that these features originate from the much smaller minigaps. For example, at $1.5 \mathrm{~K}$ (thick solid line in Fig. 2) only the minima associated with gaps separating adjacent Landau bands survive. Particularly noteworthy is the behavior of the Hall conductance [14]. It retains its quantized value of $16 e^{2} / h$ past the first miniband. As the Fermi energy is swept through the second miniband by lowering the magnetic field, the Hall conductance makes the transition to $18 e^{2} / h$ and holds this value while the third miniband is being filled. In other words, we recognize the 0-0-1-1 sequence as predicted in Ref. [9] for the left side of Hofstadter's butterfly [Figs. 1 and 3(b)]. In a homogeneous 2DES, the plateaus in the Hall conductance do not extend beyond the minima in $R_{x x}$ related to the Landau level gaps, but instead the Hall conductance gradually changes to its new value. This discrepancy with the homogeneous system is the first indication of the internal structure to the Landau bands in the modulated 2DES.

As one comes closer to the symmetry axis (peak 2), minibands still bunch into three sets (Fig. 1). However, the middle set of minibands carries substantially less weight [15] and the clear threefold splitting is lost. Concurrently, the Hall conductance no longer goes through a $0-1$ transition as the Fermi energy passes through the middle set, but rather acquires some average value in between. A similar scenario holds for peak 3, 4, and 5 , located on the right side of the butterfly. Minibands coalesce into three groups in this region as well, but as the number of states contained in the middle group is far less significant, only the outer groups manifest
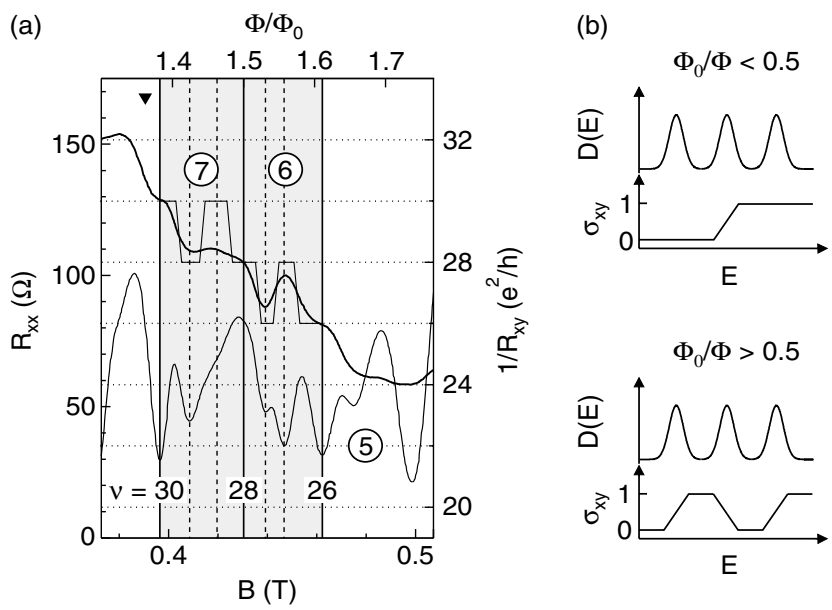

FIG. 3. (a) Expanded view of the data in Fig. 2 near peak 6 and 7 , including the schematic, idealized behavior of $1 / R_{x y}$. (b) Behavior of the Hall conductance as the energy is swept through the Landau band for the left and right side of Hofstadter's butterfly. Only the largest minigaps are considered. 


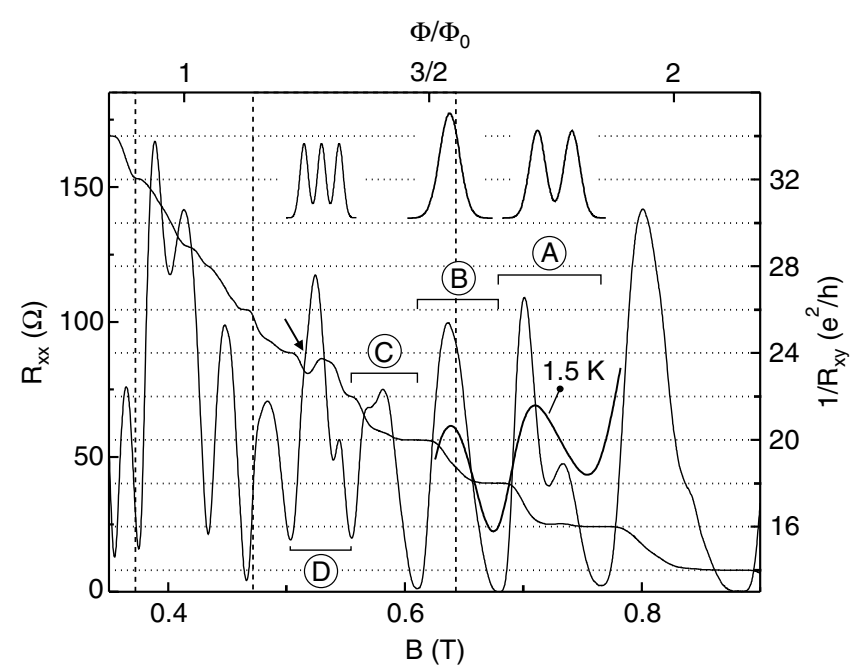

FIG. 4. The same as in Fig. 2 for the 2D-lattice with period $a=100 \mathrm{~nm}$, except that letters mark the SdH-maxima.

themselves in the experiment and cause a twofold splitting of peak 5. The flat band condition is satisfied alongside peaks 3 and 4 (dashed line in Fig. 2). Since the minigap separating the outer groups scales with the nearly vanishing width of the Landau band, the scattering-induced broadening [16] conceals the splitting (inset, Fig. 2). The evolution of the Hall conductance near peak 5 mirrors the behavior at peak 2 with one noticeable disparity: in between the plateaus, $1 / R_{x y}$ dwells closer to the quantized value on the high field side $\left(24 e^{2} / h\right)$, as opposed to our observation on the other side of the butterfly near $\phi / \phi_{0}=2$ (peak 2), where $1 / R_{x y}$ takes on a value closer to the low field plateau. This heralds the fundamental differencedespite the symmetry as far as the allowed energy states are concerned-between the left and right wing of the butterfly for $\sigma_{H}$. Other examples on the right wing, tantamount to peak 5, are features $A$ and $C$ of the $100 \mathrm{~nm}$ lattice in Fig. 4 (peak $B$ lies at the flat band condition).

Maxima 6 and 7 in Figs. 2 and 3(a), and $D$ in Fig. 4, respectively, are placed to hit the point home. Sufficiently far from the symmetry axis, the central cluster of minibands has gained a sufficient number of states. The threefold splitting ensues and the Hall conductance executes its nonmonotonous 0-1-0-1 sequence, characteristic for the right wing of the butterfly [schematically shown in Fig. 3(b)]. For peak $D$ in Fig. 4 it starts at the value $22 e^{2} / h$, jumps to nearly $24 e^{2} / h$ while the states of the lowest miniband are filled, and drops back once the second miniband passes through the Fermi energy. It does not fully recover to its starting value of $22 e^{2} / h$, and clearly the minigap is small (presumably because of the nearby zero bandwidth condition), since in $R_{x x}$ one can only discern a slope change near the arrow. After this vacillation, the Hall conductance approaches its final value $24 e^{2} / h$ as the top miniband becomes occupied. For the pair of SdH maxima 6 and 7 in Fig. 3, 1/ $R_{x y}$ traces similar nonmonotonous $0-1-0-1$ paths: unmistakably for peak 6 , but only marginally for peak 7 . The nonmonotonous Hall conductance and the splittings of the $R_{x x}$-maxima suffer from the complication that the Landau bands partly overlap at this low field. The schematic density of states diagram in the inset has only 5 peaks, instead of the expected 6. Four maxima and one shoulder structure are indeed visible in experiment. Without the analysis of $\sigma_{H}$ it would have been difficult to confidently unravel this case. Thouless' and Středa's prediction [9] is confirmed in experiment. An abundant set of other examples has been observed, and we have further elaborated and affirmed congruity by tuning the electron density $n_{s}$ (i.e., gate voltage $V_{g}$ ) while monitoring Hall and longitudinal resistance in a fixed filling factor region. The core structure of Hofstadter's butterfly has been disclosed.

We thank D. Pfannkuche and R. R. Gerhardts for fruitful discussions, and H. Gräbeldinger and M. Riek for technical assistance. This work was supported by the Bundesministerium für Bildung und Forschung (01BM622/5 and 01BM919/5).

[1] D. Hofstadter, Phys. Rev. B 14, 2239 (1976).

[2] P. G. Harper, Proc. Phys. Soc. A 68, 874 (1955).

[3] D. Langbein, Phys. Rev. 180, 633 (1969).

[4] D. J. Thouless, in The Quantum Hall Effect, edited by R. Prange and S. Girvin (Springer, New York, 1987), p. 101.

[5] N. Usov, Sov. Phys. JETP 67, 2565 (1988).

[6] U. Kuhl and H.-J. Stöckmann, Phys. Rev. Lett. 80, 3232 (1998).

[7] R. R. Gerhardts, D. Weiss, and K. von Klitzing, Phys. Rev. Lett. 62, 1173 (1989); R. W. Winkler, J. P. Kotthaus, and K. Ploog, ibid., 62, 1177 (1989); P. Vasilopoulos and F. Peeters, ibid., 63, 2120 (1989); C. Zhang and R. R. Gerhardts, Phys. Rev. B 41, 12850 (1990).

[8] V. Gudmundsson and R. R. Gerhardts, Phys. Rev. B. 52, 16744 (1995).

[9] D. J. Thouless et al., Phys. Rev. Lett. 49, 405 (1982); P. Středa, J. Phys. C 15, L1299 (1982).

[10] R. B. Laughlin, Phys. Rev. B 23, 5632 (1981).

[11] T. Schlösser et al., Europhys. Lett. 33, 683 (1996).

[12] D. Springsguth, R. Ketzmerick, and T. Geisel, Phys. Rev. B 56, 2036 (1997).

[13] C. Albrecht et al., Phys. Rev. Lett. 83, 2234 (1999).

[14] Contrary to experiment, theory delivers the Hall conductance rather than the Hall resistance. One swaps between both by inverting the conductivity or resistivity tensor. In the magnetic field regime of interest, the Hall conductance, calculated from the simultaneously measured $R_{x x}$ and $R_{x y}$ values, only marginally deviates from $1 / R_{x y}$.

[15] For example, close to the symmetry axis for $\phi / \phi_{0}=7 / 4$ the seven subbands are divided among the three clusters as follows: three belong to the top cluster, three to the bottom cluster, whereas the middle group consists of a single miniband and only contains one-third of the number of states of each of the outer clusters.

[16] D. Pfannkuche and R. R. Gerhardts, Phys. Rev. B 46, 12606 (1992). 